\title{
Zum Studium der Medizin in Basel: Die Stimme eines Studenten aus dem Jahre 1668
}

\author{
Von Leonard Forster, University College, London
}

Die Bibliothek des Französischen Spitals La Providence zu Horsham in der englischen Grafschaft Sussex enthält in einem Sammelband die Korrespondenz des Zürcher Theologen Johann Heinrich Ott (1617-1682) aus den 1660er Jahren ${ }^{1}$. Unter den Briefen an Ott befindet sich eine Reihe von Briefen von einem jungen Vetter, auch Johann Heinrich Ott geheißen, der zu Basel, Montpellier und Padua Medizin studierte. Einer von diesen Briefen bringt die Ansichten der studierenden Jugend über den Wert des Studiums der Medizin an den verschiedenen Universitäten so klar zum Ausdruck, daß er der Vergessenheit entrissen zu werden verdient. Besonders über die Universität Basel wird eine ziemlich eindeutige Sprache geführt.

Dieser junge Johann Heinrich Oтt, $1645^{2}$ geboren, studierte zuerst in Zürich Philosophie und verteidigte im Juni 1664 eine Disputation, Disquisitio physica de mutorum ac surdorum ab ortu sermone ${ }^{3}$. Im November 1665 erscheint er in der Korrespondenz zum erstenmal ${ }^{4}$; er studiert in Basel Medizin und bittet seinen Vetter um Rat über ein geeignetes Thema für eine medizinische Disputation. Er logiert mit einem Kommilitonen namens Heß, der gerade von Frankreich zurückgekommen sei und mit dem er französisch spricht; als Beweis seiner Fähigkeiten in dieser Sprache ist der Brief auch französisch geschrieben. Er fügt hinzu: «Je ne hante pas trop $\mathrm{Mr}$

\footnotetext{
${ }^{1} \mathrm{Zu}$ Johann Heinrich Ott d. Ä. vgl. Historisch-Biographisches Lexikon der Schweiz, Band V, S. 365; LEU, Schweizerisches Lexicon, Band XIV, S. $336 \mathrm{ff}$; der Briefband ist von mir in den Proceedings of the Huguenot Society of London, XIX (1953) 1 kurz beschrieben worden und Regesten der Briefe erscheinen demnächst als selbständige Veröffentlichung in den Publications derselben Gesellschaft. Mitteilungen aus der Handschrift habe ich im Euphorion XLVII (1953) $390 \mathrm{ff}$. (Briefwechsel Otts mit Philip von Zesen) und Slavonic and East European Review XXXII (1954) 475 ff. (Briefwechsel mit J.A.Comenius) gegeben. Eine Miszelle erscheint im Jahrgang 1954 der Basler Zeitschrift für Geschichte und Altertumskunde.

${ }^{2} \mathrm{Zu}$ Johann Heinrich Ott d. J. (1645-1703) vgl. C. Keller-Escher, Promptuarium genealogicum (handschriftlich auf der Zentralbibliothek Zürich); LEU, a. a. O., S. 341; er ist wahrscheinlich der Heinrich Ottius, der bei ZeDLen im Universallexicon $X X V, 2387$, als «berühmter Zürcher medicus» erscheint.

${ }^{3}$ Exemplar Universitätsbibliothek Basel.

${ }^{4}$ Ott Papers, Letters, fo. 835.
} 
Muralt, parce qu'il est un fou» (es handelt sich hier wohl um den gleichaltrigen Johannes von Muralt, 1645-1733, der 1686 das Collegium Anatomicum in Zürich eröffnen sollte ${ }^{5}$; die Rivalität mit Muralt läuft wie ein roter Faden durch die Briefe des jungen Johann Heinrich Ott. Juni 1665 hatte Muralt in Zürich über ein ähnliches Thema wie Ott disputiert - Schola mutorum et surdorum ${ }^{6}$, womit wohl die Rivalität ihren ersten Ausdruck gefunden haben mag; im Jahre vorher hatte Muralt der Disputation Otts ein freundschaftliches Carmen beigesteuert.) Im Januar 1666 ist die Frage der Disputation erledigt; das Thema heißt De Diaeta sanorum, und Ott fragt, ob er seine Zitate aus Hippokrat und Galen lateinisch oder griechisch bringen soll ${ }^{7}$. Am 14.Februar fragt er an, wem er die Disputation, die er am 26. zu verteidigen gedenkt, widmen soll ${ }^{8}$. Mit dem Dazwischenkommen der Fasnacht hatte er aber nicht gerechnet - ein gewisser Mangel an Humor scheint ihm eigen gewesen zu sein, was durch den Ton der Briefe bestätigt wird -, und er mußte die Disputation verschieben, «nisi risu excipi voluissem ». ${ }^{9}$ Tatsächlich fand sie am 9. März statt, wie aus dem Druck zu ersehen ist $^{10}$, und verlief erfolgreich. Kurz danach denkt er daran, seine Studien anderswo zu verfolgen, und denkt in erster Linie an Montpellier; mit Muralt sei es zu einem Zerwürfnis gekommen, und er beeilt sich, seinen Vetter zu versichern, daß alles, was Muralt unter Umständen über ihn erzählen könnte, jeglicher Grundlage entbehrt: «si famam meam decerpere conatus fuerit, acerbioraque de me scripserit (ut quod tantum genio indulgeam, Tabacum continuo bibam, nulla Collegia frequentem, studiis operam non navem, et si quae sunt alia) nihil cures; ipse me excusare nolo. ${ }^{11}$

${ }^{5}$ Historisch-Biographisches Lexikon der Schweiz Band V, S. 211; LEU, Lexicon, Band XIII, S. 452 ff.; C.Brunner und W.von Muralt, Aus den Briefen hervorragender Schweizer Ärzte des 17.Jahrhunderts, Basel 1919; A. HrRsch, Biographisches Lexikon der hervorragenden Ärzte, Berlin 1929-35, Band IV, S. $302 \mathrm{ff}$.

${ }^{6}$ Exemplar Universitätsbibliothek Basel.

${ }^{7}$ Ott Papers, Letters, fo. 839.

${ }^{8}$ Ebenda, fo. 843.

9 «Wenn ich mich nicht dem Gespött der Leute aussetzen wollte», ebenda, fo. 845 .

${ }^{10}$ Basileae, typis Joh. Jac. Deckeri; Exemplar Universitätsbibliothek Basel. Die Zitate aus griechischen Autoren erscheinen in der Ursprache.

11 «Beachten Sie es nicht, wenn er versuchen sollte, mich um meinen guten Ruf zu bringen und wenn er einiges Schlimmes über mich schreiben sollte, zum Beispiel, daß ich nur meinem Vergnügen nachgehe, ständig Tabak rauche, keine Vorlesungen besuche, keine Mühe auf das Studium verwende und so weiter; ich selbst habe keine Absicht, mich zu entschuldigen» ebenda, fo. 849. 
Im September finden wir ihn in Montpellier, wo er gut arbeiten kann und jeden Tag ins «Nosodochium» geht ${ }^{12}$. Im Juni $1667^{13}$ berichtet er, er habe im vergangenen Jahr den Plan gehegt, sein Studium nach Paris zu verlegen, jetzt aber denke er ernsthaft an Padua, weil ihm sein Freund Hegnerus von dort begeistert über das Studium der chirurgischen Fächer in Padua geschrieben habe. «Scribit enim in illa Universitate studium Anatomicum et Chirurgicum quàm maximè florere, tempore hyberno in Nosocomio (quod cuilibet quolibet tempore adire liceat) omnes operationes Chirurgicas praemisso discursu in cadaveribus humanis demonstrari.» ${ }^{14}$ Vier Tage später aber plagt ihn der Zweifel, ob in Padua das chemische Studium auch so blühe; er habe sich nämlich ganz der Chemie ergeben. «Ratio autem cur Chymiam tanti aestimem est, quòd videam praestantissimos ex Chymicis remediis (il nostris praesertim regionibus magis septentrionalibus) in medicina effectus, neque tanti constent quanti Galenica: accedit aemulatio, cum enim Muraltus Lugd. Bat. degens, optimam Chymiam addiscendi habeat occasionem, nisi eandem Patavij haberem occasionem, aemulatio me quasi consumeret. » ${ }^{15}$ Seine Zweifel scheinen sich gelegt zu haben, denn der nächste Brief fältt ein Jahr später - aus Padua; er kommt unten zum Abdruck.

Die beiden letzten Briefe ${ }^{16}$ sind 1668 und 1669 geschrieben, offenbar nach der Promotion, denn er zeichnet am 30. November 1668: Johannes Henricus Ottius Philosoph. et Medic. Doctor; sie handeln von der Verlängerung seines Stipendiums und den Möglichkeiten einer Reise nach Dalmatien und brauchen uns in diesem Zusammenhang nicht weiter zu beschäftigen.

[Ott Papers, Letters, fo. 859.]

12 Ebenda, fo. 851.

13 Ebenda, fo. 855.

14 «Er schreibt mir nämlich, daß an jener Universität das Studium der Anatomie und der Chirurgie am prächtigsten im Winter gedeiht und daß im Krankenhaus (das jeder zu jeder Zeit betreten darf) alle chirurgischen Operationen nach einem einleitenden Vortrag an menschlichen Leichen demonstriert werden.» Hans Heinrich Hegner promovierte 1668 in Padua Dr. med.; LeU, Lexicon, Band X, S. 20.

15 «Der Grund, weshalb das ich Studium der Chemie so schätze, besteht darin, daß ich die außerordentlichsten Wirkungen in der Medizin (besonders in unseren nördlicher gelegenen Gegenden) aus chemischen Heilmitteln hervorgehen sehe, auch kosten sie nicht so viel wie die des Galen; dazu kommt der Neid: da nämlich Muralt, der in Leiden ist, die beste Gelegenheit hat, die Chemie zu lernen, würde mich geradezu der Neid fressen, wenn ich nicht in Padua eine ebensogute Gelegenheit haben sollte», Ott Papers, Letters, fo. 857 .

${ }^{16}$ Ebenda, fo. 865 und 869. 


\section{Vir admodum Reverende}

\section{$S P$}

Ex ultimis, quas Parens ad me dedit, litteras percepi, tibi non probari quòd Patavij doctoralem gradum accipere contendam, eò quòd Doctores Patavij creati non in tanto sint aestimio, quanto Doctores Basileae creati. Cùm autem haec non nisi ex paterno animo profecta sciam, ut meo officio satisfaciam, Reverentie, tuae in praesentibus meas quae me ad hanc rem impulerunt rationes, proponam, non dubitans, quin mecum in eandem sententiam sis conscensurus. Dico itaque quòd locus ubi quis Doctor creatur, nihil facit ad rei essentiam, sed meré accidentale ${ }^{17}$ quid est, et consequenter Doctorem neque doctiorem, neque indoctiorem arguit. Verum quidem est quòd nonnunquam Patavij Doctores creentur, qui in studio medico non admodum excellunt; sed etiam hoc verum est, quòd Medici hodierno die celeberrimi et experientissimi totius Germanie, (ut Rolfinckius Schenckius, Welschius, Michaël, Schroëderus, Zwelferus, Bartholinus ${ }^{18}$ et innumeri alij) hic Patavij Doctores promoti fuerint. Itaque non bona est haec consequentia; Patavij Doctor creatus est; Ergo est indoctus. Sed quaeso ubinam hodierno tempore locus est, ubi non omnis generis (id est docti aequé ac indocti) Doctores creentur? Monspelij ante aliquot annos adhuc nullus, nisi doctissimus fuerit, ad doctoratum fuit admissus, jam verò docti aequé ac indocti admittuntur. Quales Lugduni Batavorum, Jene, etc (academiis tamen celeberrimis, et ubi rigorosé satis proceditur) Doctores creentur notum est. Ubique auri sacra fames ${ }^{19}$ multùm efficit. Neque verò Basileae res se melius habet; cùm enim ibi degerem, duo studiosi promoti fuêre, qui adeò indocti erant, ut omnes studiosi admirati fuerint, et invicem dixerimus, quòd nullus possit dubitare quin admittatur, postquam illi duo admissi fuerint.

17 Man beachte die steife, formallogische Beweisführung in diesem Briefe, zu welcher die scholastischen termini «essentia» und «accidentale» gut stimmen; der junge Mann will seine philosophische Vorbildung im besten Lichte zeigen.

18 Werner Rolfinck (1599-1673), Allgemeine Deutsche Biographie, Band XXIX, S. 74, Hrrsch, a.a.O. Band IV, S. 861; Joh. Theod. Schenckius (1619-1671), ADB, Band XXXI, S. 51, Hırsch, Band V, S. 64; Georg Hieronymus Welsch (1624-1677), ADB Band XLI, S. 681; Johann Michaelis (1606-1667), HIrsch, Band IV, S. 197; Johannes Schroederus (1600-1664), ADB, Band XXXV, S. 518, Hirsch, Band V, S. 140; Johannes Zwölferus (1618-1668), HIrsch, Band V, S. 1055; Thomas Bartholinus (1616-1680), C.F.BrickA, P.Engelstoft und Sv.DAHL, Dansk Biografisk Leksikon, Kopenhagen 1933-44, Band II, S. 205 ff., Hrrsch, Band V, S. 250 ff. - Aus dieser Liste promovierten Rolfinck und Zwölfer tatsächlich in Padua; die anderen hielten sich allerdings längere Zeit dort auf, promovierten jedoch anderswo, Welsch und Bartholinus sogar in Basel! 19 Äneis III. 56. 
Antequam Basileam venissem, ejusdem farine, promoti fuêre. Post meum discessum tam miser Doctor creatus est, ut ne quidem disputationem pro gradu suscipere ausus fuerit sed pro disputatione ter publicé legere coactus fuerit. Si quis Basilee, erga Professores liberalem se exhibeat, lectionibus eorum diligenter intersit, post lectiones eos domum comitetur, et in aliis quoque occasionibus eos magno honore afficiat, infallibiliter, sive doctus sive indoctus fuerit, ad doctoratum admittitur! Itaque neque valet haec argumentatio; Basileae Doctor creatus est; Ergo est doctus; ergo debet plus aestimari quàm alius. Praeterea cùm, cùm Tiguri adhuc degerem, semper diligens habitus fuerim et forsan etiamnum ab aliquibus talis existimer, non facilé credent me ob ignorantiam, et quòd Basilee, non admissus fuissem, hic Patavij doctoralem gradum accepisse. Tandem si quid de sumptibus loquendum est, Patavij totus actus doctoralis, cum omnibus accidentibus, plus quàm sexaginta taleris non constat, cùm tamen si Basileae Doctor promoveri vellem, adhuc ducentos taleros ad minimum insumere deberem, prout Parenti prolixé satis, et specificativé (ut ita loquar) perscripsi. Et haec quidem, Vir praeclarissime, sunt meae rationes, si tuum rescribere placeat judicium, gratissimum erit. Quia autem in hac Universitate non moris est disputare pro gradu, neque ego disputationem suscipiam, quam Amplissimo Magistratui dedicare possim. Quod ad stipendium attinet, nescio quo modo sit procedendum, ut mihi ulterius erogetur, quare et hac de re tuum consilium proximé expecto. Quod iter per Italiam peragere non potuerim, vehemnter doleo. Sequente septimana D. Hegenerus Doctor creabitur; et ipse domum redire cogitur. Fama spargitur Tiguri pestem grassari. Hisce, Vir praeclarissime, vale, meque porrò amare perge.

Reverentiae tue, addictissimus, et ad omnia officia promptissimus

Patavij die 19 Julij 1668

$$
\text { Johannes Henricus Ottius }
$$

Si placet, meo nomine salute plurima impertias dilectissimam uxorem tuam, D. Profess. Lavaterum, et eloquentissimum D. Schaedlerum ${ }^{20}$.

Monsieur

Monsieur Ott, Professeur en eloquence

Zurig.

${ }^{20}$ Professor Lavater ist wohl Johannes Lavater (1624-1695), der 1657 Professor der Rhetorik und Logik am Collegium Humanitatis in Zürich wurde und sich besonders mit dem Problem der Taubstummenbildung beschäftigte, Historisch-Biographisches Lexikon der Schweiz, Band IV, S. 636. Von ihm hatte Oтт das Thema zu seiner philosophischen Disputation. Johann Jakob Schaedler (1613-1676), ein Anverwandter Johann Heinrich Otts d. Ä., war seit 1663 Dekan des oberen Thurgauer Kapitels, HistorischBiographisches Lexikon der Schweiz, Band VI, S. 114. 


\section{Hochverehrter Herr, ${ }^{21}$}

Unter den letzten Briefen von meinem Vater erhielt ich einen, aus dem ich erfahre, daß Sie nicht damit einverstanden sind, daß ich mich bemühe, in Padua den Doktorgrad zu erringen, weil die in Padua gemachten Doktoren nicht so geschätzt werden wie die in Basel gemachten. Da ich aber weiß, daß dies nur aus dem Kopfe meines Vaters stammt, möchte ich Ihnen, verehrter Herr, gerne pflichtschuldigst in diesem Briefe die Gründe, die mich zu diesem Unterfangen antrieben, erklären, ohne zu zweifeln, daß Sie mit mir in diesem Ratschluß übereinstimmen werden. Ich sage also, daß der Ort, wo jemand zum Doktor gemacht wird, nichts Wesentliches ist, sondern nur etwas Zufälliges, und daher weder auf einen gelehrteren noch einen ungelehrteren Doktor schließen läßt. Es ist zwar wahr, daß manchmal in Padua Doktoren gemacht werden, die im medizinischen Fach nicht gerade hervorragend sind; aber auch das ist wahr, daß alle heute berühmtesten und erfahrensten Ärzte von ganz Deutschland (wie Rolfinck, Schenck, Welsch, Michaëlis, Schroëder, Zwelfer, Bartholin und zahllose andere) hier in Padua zum Doktor promoviert wurden. Daher ist es falsch, den Schluß zu ziehen: Er wurde in Padua zum Doktor gemacht; also ist er ungelehrt. Sondern ich frage, wo es heutzutage einen Ort gibt, wo nicht alle Arten von Doktoren (will sagen gelehrte ebenso wie ungelehrte) gemacht werden? Bis vor einigen Jahren wurde in Montpellier niemand zum Doktorat zugelassen, der nicht höchstgelehrt war, aber jetzt werden Gelehrte und Ungelehrte zugelassen. Es ist wohlbekannt, was für Doktoren in Leiden, Jena usw. (die doch gewiß berühmte Universitäten sind, wo genügend streng vorgegangen wird), gemacht werden. Überall übt die verwünschte Sucht nach Gold ihre Macht aus. Nicht einmal in Basel verhält sich die Sache besser; denn als ich dort wohnte, wurden zwei Studenten promoviert, die solche Nichtswisser waren, daß sich alle Studenten verwunderten, und wir einer zum andern sagten, daß niemand zweifeln könne, ob er zugelassen wird, nachdem diese zwei zugelassen wurden. Leute von der gleichen Qualität wurden dort promoviert, bevor ich nach Basel kam. Nach meiner Abreise wurde ein so miserabler Doktor gemacht, daß er es nicht wagte, eine Disputation für seinen Doktorgrad zu halten, sondern anstatt der Disputation gezwungen war, dreimal öffentlich vorzulesen. Wenn jemand sich in Basel freigebig gegen die Professoren erweist, eifrig ihren Vorlesungen bei-

${ }^{21}$ Die Übersetzungen der lateinischen Stellen im Text dieses Beitrags sowie die Verdeutschung des Briefes sind von Marianne Winder, der ich an dieser Stelle meinen Dank aussprechen möchte. 
wohnt, sie nach den Vorlesungen nach Hause begleitet und ihnen auch bei anderen Gelegenheiten große Ehren antut, wird er unbedingt zum Doktorat zugelassen, ob er nun gelehrt oder ungelehrt ist. Daher trifft die folgende Erwägung nicht zu: Er wurde in Basel zum Doktor gemacht; daher ist er gelehrt; daher muß er mehr geschätzt werden als ein anderer. Außerdem werden die Leute nicht leicht glauben, daß ich aus Unwissenheit und weil ich in Basel nicht angenommen wurde, hier in Padua den Doktorgrad erhalten habe, da ich bisher, während ich in Zürich lebte, immer gewohnheitsmäßig fleißig war und wahrscheinlich auch von anderen als so angesehen werde. Außerdem, um auf den Geldaufwand zu sprechen zu kommen, kostet in Padua die ganze feierliche Handlung mit allem Drum und Dran nicht mehr als sechzig Taler, während, wenn ich in Basel zum Doktor promovieren wollte, ich dazu mindestens zweihundert Taler in Anspruch nehmen müßte, wie ich meinem Vater lang und breit genügend eindringlich und sozusagen mit allen Einzelheiten geschrieben habe. Und dies sind nun meine Gründe, weitberühmter Herr. Ihr Urteil in einem Antwortschreiben wäre mir sehr willkommen. Da es aber an dieser Universität nicht Sitte ist, um einen Doktorgrad zu disputieren, werde ich auch keine Disputation unternehmen, die ich dem Ehrwürdigen Rat widmen könnte. Bezüglich des Stipendiums weiß ich nicht, auf welche Art man vorzugehen hat, damit einem mehr ausgezahlt wird, weshalb ich in dieser Angelegenheit Ihren Rat so bald wie möglich erhoffe. Es tut mir außerordentlich leid, daß ich meine Reise durch Italien nicht unternehmen kann. Nächste Woche wird Herr Hegner zum Doktor gemacht; auch er ist gezwungen, nach Hause zurückzufahren. Es geht das Gerücht um, daß in Zürich die Pest grassiert. Hiermit, berühmtester Mann, leben Sie wohl, und haben Sie mich auch in Zukunft weiter gern.

In Verehrung,

Ihr ergebenster und zu allen Diensten bereiter

Johann Heinrich Ott

Padua, am 19.Juli 1668

Bitte, richten Sie Ihrer lieben Gattin, dem Herrn Professor Lavater und dem so beredten Herrn Schaedler meine herzlichsten Grüße aus. 